\title{
The impact of repeated prostate biopsies on sexual function and urinary symptoms in patients with diagnosis of Atypical Small Acinar Proliferation (ASAP): Can ecoDoppler reduce side effects?
}

\author{
Luigi Quaresima, Vito Lacetera, Luca Leone, Lorenzo Montesi, Ubaldo Cantoro, \\ Massimo Polito, Giovanni Muzzonigro \\ Institute of Urology, AOU United Hospitals, Polytecnic University of Marche Region, Ancona, Italy.
}

\begin{abstract}
Summary
Objectives: To establish whether repeated trans-rectal ultrasound-guided Prostate Needle Biopsies (PNBX) performed in men with diagnosis of Small Acinar Atypical Proliferation (ASAP) predispose these subjects to Erectile Dysfunction (ED) and to evaluate if EcoColorDoppler (ECD) can help to reduce this side effect.

Materials and methods: We performed a retrospective study regarding 190 men with diagnosis of ASAP detected between January 2001 and December 2011, who underwent to repeated prostate needle biopsies $(\mathrm{PNBX})$. These patients were investigated about Erectile Function (EF) and Lower Urinary tract Symptoms (LUTS) using International Index of Erectile Function (IIEF-5) and International Prostate Symptom Score (IPSS) questionnaires before the first PNBX and 3 months after each other one. In particular, among the 89 men without ED before first PNBX, we compared IIEF-5 score between 64 patients who underwent to standard PNBx and 25 patients submitted to a PNBx done with in addition ECD ultrasound imaging. Results: Mean patient age was 65 years (SD 7.7); mean follow-up was 3.2 years (SD 1.8) and the mean number of re-biopsies completed was 2 (SD 1.5). Among the 143 men considered, only 89 resulted with a normal EF (IIEF-5 score > 21): in this group incidence of ED (IIEF-5 score $<21$ ) among patients who underwent to standard PNBx was 4/64 (6.25\%) while in patients submitted to a $P N B x$ with ECD was 1/25 (4\%). A greater decrease of EF was observed in patients undergone to 3 or more biopsies; no relationship between IPSS score and re-PNBx was identified.

Conclusion: Repeated PNBX done in patients with diagnosis of ASAP appear to get worse EF; number of biopsies seems to increase the risk of ED. Use of ECD in transrectal ultrasound-guided PNBX may have a role to avoid neurovascular bundles (NVBs) and preserve EF; anyway further studies are highly recommended to validate this hypothesis.
\end{abstract}

KEY WORDS: Prostate biopsy; Erectile dysfunction; Small Acinar Atypical Proliferation (ASAP).

Submitted 3 October 2014; Accepted 31 October 2014

\section{INTRODUCTION}

Trans-rectal ultrasound-guided Prostate Needle Biopsy (PNBx) is the diagnostic practice of choice for prostate cancer ( $\mathrm{PCa}$ ) detection; it is considered a safe and routinely procedure, with a low risk of major complications requiring hospital admission (1), as fever and sepsis. Minor complications, such as rectal bleeding, acute urinary retention, hematospermia and hematuria are relatively frequent but usually self-limiting $(2,3)$. Besides this well-documented side-effects, an increasing number of articles is showing a possible role of PNBx to cause Erectile Dysfunction (ED) (4-6). Some studies described this effect as temporary (7-9), and attributed to anxiety (9) rather than a real organic injury (6). Moreover, this studies often investigated Erectile Function (EF) in patients on active surveillance, that is to say with a diagnosis of prostate cancer, a potential negative psychological confounder that may influence their EF (10).

Another cohort of men who underwent to seriate PNBx is that of patients with diagnosis of Atypical small acinar proliferation (ASAP), a kind of precancerous lesion considered to be an element in the progression of changes in cell morphology between healthy tissue of the prostate and PCa (11). Furthermore, due to the opinion that at least some cases of ASAP diagnosis in biopsy specimens are actually undiagnosed cancers (12), the indication to second biopsy in such patients has been commonly accepted $(11,13)$.

To the best of our knowledge, no study has investigated the impact on EF of seriate PNBx done in patients with diagnosis of ASAP; in the current article, we analyzed this aspect and evaluated if ecocolorDoppler can help to prevent this side effect (Figure 1).

\section{MATERIALS AND METHOdS}

This retrospective study includes 190 men with a diagnosis of ASAP detected between January 2001 and December 2011 and undergone to repeated PNBx.

Baseline EF was evaluated before the first biopsy using 
Figure 1.

Neurovascular bundles detected by ECD imaging.

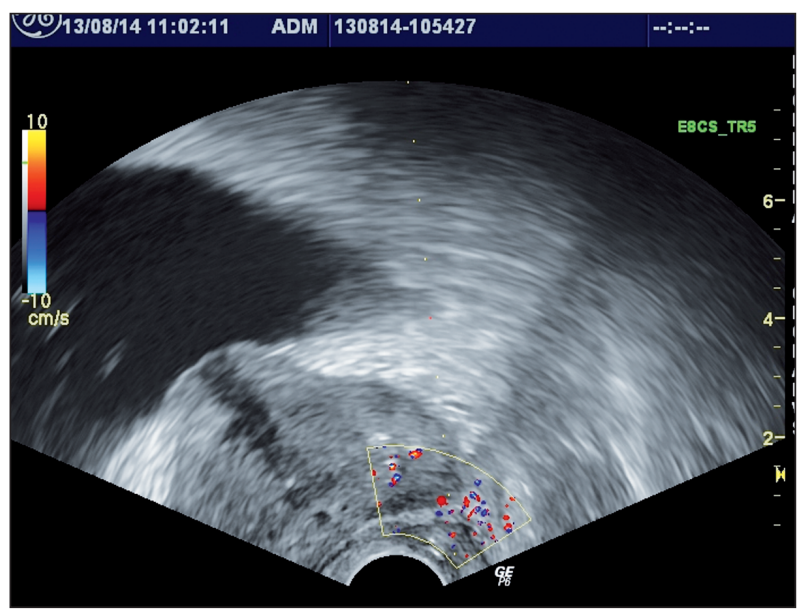

International Index of Erectile Function (IIEF-5) questionnaire; by the obtained score the patients were stratified in 5 groups (Figure 2). According to the literature, patients belonging to Group 1 (defined by an IIEF-5 score $>21$ ), were considered having a normal EF. In order to complete the study of the patients it was also investigated the preexistence of Lower Urinary Tract Symptoms (LUTS) by using International Prostate Symptom Score (IPSS). Regarding the procedure, after quinolone chemoprophylaxis and local anesthesia, it was managed a 12-16 core transrectal biopsy using a Tru Cut Cook 18G needle under transrectal uiltrasound (TRUS) control. In particular, in the group 1 of 89 patients (Figure 2) not affected by ED (defined by an IIEF-5 score $<22$ ) before the first PNBx, 64 patients underwent to standard transrectal biopsies; whereas in a subgroup of 25 Power Doppler evaluation was added to the standard ultrasonography imaging to better identify neurovascular bundles (NVBs) and avoid them with the needle.

Figure 2.

Patients stratified in 5 groups by baseline IIEF-5.

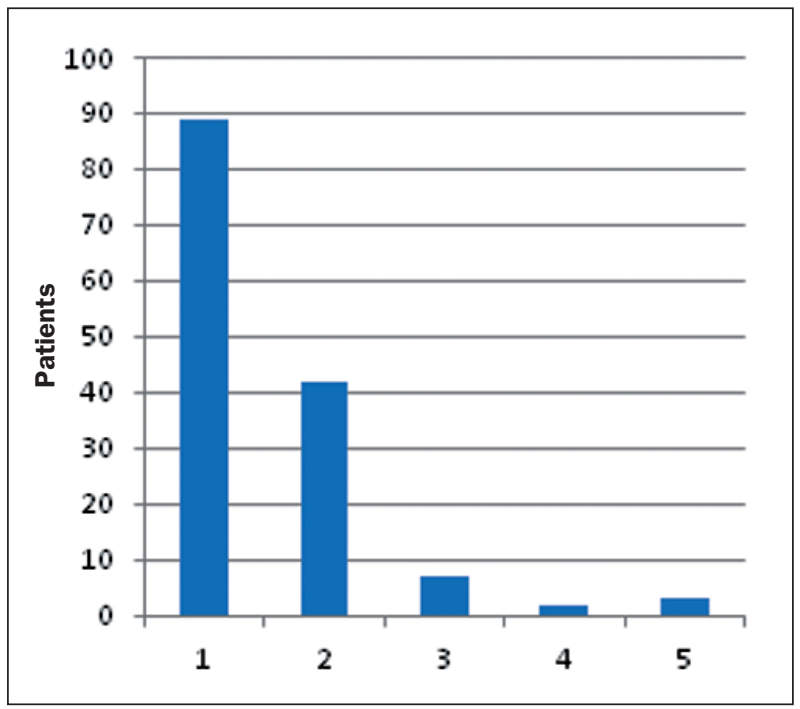

A comparison of IIEF-5 and IPSS score submitted before and 3 months after PNBx was performed to analyze differences between baseline and follow-up IIEF-5 scores, and eventually the relation with the number of PNBx done. T Student's test was used; a $\mathrm{p}$ value $<0.05$ was considered statistically significant.

\section{REsults}

Patients who were analyzed in this study resulted to have a mean age of 65 years (SD 7.7); their mean follow-up was 3,2 years (SD 1.8) and the mean number of re-biopsies was 2 (SD 1.5). Among the 143 men who concluded the follow up, only 89 resulted with a normal EF (corresponding to an IIEF-5 score $>21$ ): in this group incidence of ED (IIEF-5 score < 21) among patients who underwent to standard PNBx was 4/64 (6.25\%) while in patients submitted to a PNBx with ECD was $1 / 25$ (4\%). Univariate analysis was performed to compare the variation in IIEF-5 score (considered as the difference between the baseline IIEF and the IIEF-5 at the last follow up) in men without previous ED who underwent to repeated PNBx: the $\mathrm{p}$ value for this difference was $<0.001$, showing a significant correlation between repeated PNBx and EF as illustrated in Figure 3.

Figure 3.

Change of IIEF-5 score in patients

without previous ED (group I, $p<0.001$ ).

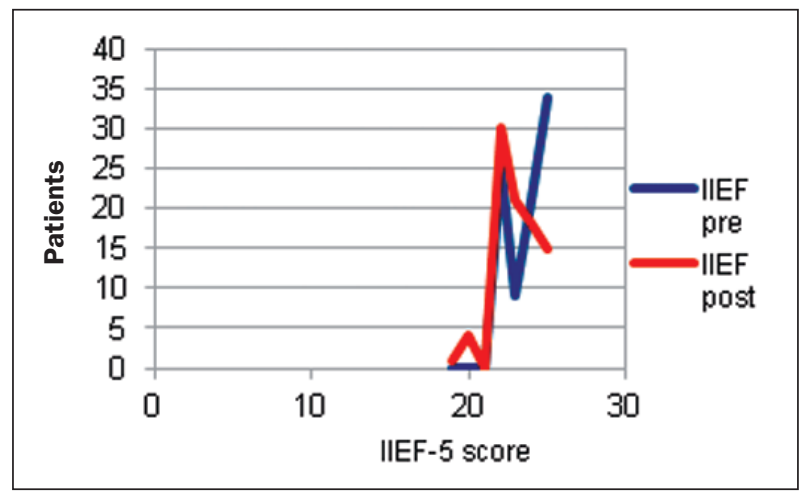

Moreover, the variation of the IIEF-5 score in patients who underwent 0 to 2 re-PNBx resulted significantly lower than in men who underwent more than 2 re-PNBx $(\mathrm{p}<0.05)$, demonstrating a relationship with the number of PNBx done. On the other hand, no significant worsening of IPSS score was identified after re-PNBx, with only temporary irritative urinary symptoms reported by some patients.

\section{Discussion}

So far is not clearly defined by the international literature whether serial PNBx predispose to a permanent ED over time: nevertheless only few studies, as the one leaded by Hilton et al., showed a total absence of correlation between this side effect and the procedure (14). In the majority of the examined studies, the investigators demonstrated a short-term (30 days) impact on EF, asso- 
ciated to psychological factors as anxiety and diagnosis of PCa $(8,10)$ rather than to use of periprostatic nerve block and flogosis linked to the procedure. Some authors considered PNBx follow-up at 1 and 6 months: Akbal et al showed a worsened EF 1 month after PNBx that returns at baseline level at 6 months (3): in this study the authors analyzed specifically only PCa-free patients, avoiding bias due to the cancer detection. Tuncel et al. described an high number of patients (up to $41 \%$ ) without ED that developed a deterioration of EF one month after PNBx: 6 months later 15\% still had ED, demonstrating a significant correlation between PNBx and ED (4). Recently, another article of Tuncel showed that the association between PNBx and DE might have an organic basis, demonstrating with power Doppler ultrasonography an inflammation of the prostate parenchyma and of the surrounding neurovascular bundle in patients suffering from ED after PNBx (6). This last article, may give a possible explication of the utility, described in our study, to add Power Doppler to the standard ultrasonography imaging, to better identify neurovascular bundles (NVBs), and avoid them with the needle. This care towards the bundles has become nowadays more and more important in consideration to the increase of the number of cores taken in each PNBx such as the practice of submit patients to repeated PNBx.

\section{Conclusions}

Men with diagnosis of ASAP who uunderwent to repeated PNBx are at risk of worsening EF; this risk appears to grow with the increase of biopsy number. Furthermore, this study proposes the use of ECD in transrectal ultrasoundguided PNBx with the purpose to avoid neurovascular bundles (NVBs) and preserve EF; nevertheless additional studies are highly suggested to validate this hypothesis.

\section{ACKNOWLEDGMENTS}

We thank Prof. Stefano Bompadre for the guidance supplied.

\section{References}

1. Pinkhasov GI, Lin YK, Palmerola R, et al. Complications following prostate needle biopsy requiring or emergency department visits-experience from 1000 consecutive cases. BJU Int. 2012; 110:369-374.

2. Djavan B, Waldert M, Zlotta A, et al. Safety and morbidity of first and repeat transrectal ultrasound guided prostate needle biopsies: results of a prospective European prostate cancer detection study. J Urol. 2001; 166:856.

3. Akbal C, Turker P, Tavukcu HH, et al. Erectile function in prostate cancer-free patients who underwent prostate saturation biopsy. Eur Urol. 2008; 53:540.

4. Tuncel A, Kirilmaz U, Nalcacioglu V, et al. The impact of transrectal prostate needle biopsy on sexuality in men and their female partners. Urology. 2008; 71:1128-31.

5. Fujita K, Landis P, McNeil BK, et al. Serial Prostate biopsies are associated with an increase risk of erectile dysfunction in men with prostate cancer on active surveillance. J Urol. 2009; 182:2664-9.
6. Tuncel A, Toprak U, Balci $M$, et al. Impact of transrectal prostate needle biopsy on erectile function: results of power Doppler ultrasonography of the prostate. Kaohsiung J of Med Sciences. 2014; 30:194-199.

7. Chrisofos M, Papatsoris AG, Dellis A, et al. Can prostate biopsies affect erectile function? Andrologia. 2006; 38:79-83.

8. Pepe P, Pietropaolo F, Dibenedetto G, et al. Erectile function after repeat saturation prostate biopsy: our experience in 100 patients. Arch Ital Urol Androl. 2013; 85:130-132.

9. Glazer AP, Novakic K, Helfand BT. The impact of prostate biopsy on urinary symptoms, erectile function, and anxiety. Curr Urol Rep. 2012; 13:447-454

10. Helfand BT, Glaser AP, Rimar K, et al. Prostate cancer diagnosis is associated with an increased risk of erectile dysfunction after prostate biopsy. BJU Int. 2013; 111:38-43.

11. Adamczyk P, wolski Z, Butkiewicz R, et al. Significance of atypical small acinar proliferation and extensive high-grade prostatic intraepithelial neoplasm in clinical practice. Cent European J Urol. 2014; 67:136-141.

12. Mazzucchelli R, Colanzi P, Pomante R, et al. Prostate tissue and serum markers. Adv clin Path. 2000; 4:111-20.

13. Iczkowski KA, MacLennan GT, Bostwick DG. Atypical small acinar proliferation suspicious for malignancy in prostate needle biopsies: clinical significance in 33 cases. Am J Surg Pathol. 1997; 21:1489-1495.

14. Hilton JF, Blaschko SD, Whitson JM, et al. The impact of serial prostate biopsies on sexual function in men on active surveillance for prostate cancer. J Urol. 2012; 188:1252-1258.

\author{
Correspondence \\ Luigi Quaresima, MD (Corresponding Author) \\ luigiquaresima@yahoo.it \\ Vito Lacetera, MD \\ vlacetera@gmail.com \\ Luca Leone, MD \\ lucaleone@yahoo.it \\ Lorenzo Montesi, MD \\ lorenzomontesi@yahoo.it \\ Ubaldo Cantoro, MD \\ ubymaior@libero.it \\ Massimo Polito, MD \\ max_polito@virgilio.it \\ Giovanni Muzzonigro, MD \\ g.muzzonigro@univpm.it
}

Institute of Urology, AOU United Hospitals,

Polytecnic University of Marche Region, Ancona, Italy 